\title{
A Single Injection of Pregabalin Induces Short- and Long-Term Beneficial Effects on Fear Memory and Anxiety-Like Behavior in Rats with Experimental Type-1 Diabetes Mellitus.
}

Alvaro Henrique Bernardo de Lima Silva

UFPR: Universidade Federal do Parana

Debora Rasec Radulski

UFPR: Universidade Federal do Parana

Gabriela Pereira

UFPR: Universidade Federal do Parana

Alexandra Acco

UFPR: Universidade Federal do Parana

Janaína Menezes Zanoveli ( $\boldsymbol{\sim}$ janaina.zanoveli@ufpr.br)

Universidade Federal do Parana

\section{Research Article}

Keywords: Streptozotocin, Post-traumatic stress disorder, elevated plus maze, contextual conditioned fear, oxidative stress.

Posted Date: November 12th, 2021

DOI: https://doi.org/10.21203/rs.3.rs-1053770/v1

License: (c) (i) This work is licensed under a Creative Commons Attribution 4.0 International License.

Read Full License 


\section{Abstract}

Anxiety Disorders and Posttraumatic Stress Disorders (PTSD) associated with type 1 diabetes mellitus (DM1) are increasingly common comorbidities and the treatment is quite challenging. In that sense, evidence indicates that the anticonvulsant pregabalin is highly effective in treating severe cases of anxiety, as well as PTSD and diabetic neuropathic pain which is also very prevalent in T1DM. Herein, the short- and long-term effects of a single injection of pregabalin on the acquisition of a fear extinction memory and parameters of anxiety in induced-T1DM animals were investigated. For that, we used the contextual fear conditioning (CFC) and elevated plus maze (EPM) paradigms, respectively. A putative antioxidant activity was also evaluated. Our findings demonstrated that induced-T1DM animals presented greater expression of fear memory, difficulty in extinguishing this fear memory, associated with a more pronounced anxiety-like response. Pregabalin was able to induce a short and long-lasting effect by facilitating the acquisition of the fear extinction memory and inducing a later anxiolytic-like effect. Also, the increased lipid peroxidation levels in the hippocampus and prefrontal cortex of induced-T1DM rats were reduced after pregabalin injection, while the decreased levels of reduced glutathione were increased in the hippocampus. Despite the need for more studies to understand the mechanism of action of pregabalin under these conditions, our data demonstrate for the first time that a single injection of pregabalin in a specific time window was able to improve behavioral parameters in addition to inducing neuroprotective effect. Thus, pregabalin has potential worth exploring for the treatment of PTSD and/or Anxiety associated with T1DM.

\section{Introduction}

Accumulating evidence indicates that diabetic encephalopathy and psychopathologies share common neurobiological mechanisms (de Morais et al. 2014; Gupta et al. 2014; Prabhakar et al. 2015; da Silva Dias et al. 2016; Aswar et al. 2017; Chaves et al. 2020). In this sense, preclinical studies conducted in diabetic or non-diabetic animals show that increase in inflammation and oxidative stress relatedprocesses, along with a dysregulation in neurotransmission systems in brain areas related to the emotions, like the hippocampus (HIP) and prefrontal cortex (PFC), are present in animals with anxiety and/or depressive-like behaviors (MacQueen et al. 2005; de Morais et al. 2014; Muriach et al. 2014; Moulton et al. 2015; Bludau et al. 2016; Buchberger et al. 2016; da Silva Dias et al. 2016; Zanoveli et al. 2016; Pereira et al. 2018; Banagozar Mohammadi et al. 2019; Chaves et al. 2020).

Because these brain areas are highly involved in the processing of learning and memory, it is not surprising that clinical studies demonstrate cognitive dysfunctions in diabetic patients along with cortical and HIP atrophy and white matter lesions (Bremner et al. 1995). Here, it is important to highlight that diabetic patients are more likely than non-diabetic individuals to experience cognitive dysfunction along with anxiety (Jacobson et al. 2011; dos Santos et al. 2014; Rajput et al. 2016). Also, studies point out that post-traumatic stress disorder (PTSD), a disorder characterized by aberrant consolidated fear memories and/or failure in extinguishing fear memory, is a risk factor for the development of T1DM and type-2 diabetes mellitus (Weisberg et al. 2002; Zung et al. 2012; Vaccarino et al. 2014; Renna et al. 2016). 
Preclinical studies reinforce these clinical findings, by demonstrating that animals with experimental type1 diabetes mellitus (T1DM) present learning and memory impairments (Matsunaga et al. 2016; Ahmed et al. 2019). This impairment is also extended when studies turn to fear memory processing (lkeda et al. 2015; Gambeta et al. 2016; de Souza et al. 2019; Ribeiro et al. 2020). Thus, our group showed that induced-T1DM animals present an overconsolidation of fear memory coupled with a generalization of this fear response when they are exposed to a neutral (non-aversive) environment (Gambeta et al. 2016; de Souza et al. 2019). Furthermore, they also have difficulty in extinguishing a fear memory (Ribeiro et al. 2020).

The association of these diseases considerably impairs the quality of life of patients, in addition to facilitating the emergence of other comorbidities and causing a great economic impact on society. Also, treating PTSD associated with T1DM with first-line drugs - antidepressant drugs - is a huge challenge (Lustman e Clouse 2005; for a review see Zanoveli et al. 2016). Thus, exposure psychotherapy has been applied to contribute to the development of a fear/trauma extinction memory and it has become a valuable and safer treatment. Bearing in mind that extinction memory means new learning capable of inhibiting the expression of fear memory, the search for drugs that facilitates extinction processes without impairing the glycemic control and/or body weight would be excellent candidates for the treatment of PTSD particularly in individuals with T1DM.

In this respect, it is known that the anticonvulsant pregabalin (PGB), a gabapentin derivative of gammaaminobutyric acid, has been used to treat severe forms of generalized anxiety disorder (GAD) and also PTSD (Pande et al. 2003; Baniasadi et al. 2014; Greenblatt e Greenblatt 2018) and it has shown promising results as a potential therapeutic alternative. Considering particularly the diabetic condition, it is noteworthy that PGB presents a broad-spectrum efficacy in the treatment of diabetic neuropathic pain (Finnerup et al. 2015), a condition that affects more than 25\% of diabetic patients (Tesfaye et al. 2013; Finnerup et al. 2015). Thus, from this perspective, treating PTSD associated with T1DM with PGB would be advantageous. The exact mechanisms of action of PGB are still not completely elucidated; however, studies in vitro demonstrate that the drug binds presynaptic to the protein subunit (a2- $\delta$ ) of voltagedependent calcium channels in the central nervous system (Rickels et al. 2005), thereby suppressing the release of excitatory neurotransmitters, including glutamate, substance $P$ and noradrenaline (Field et al. 2001; Taylor et al. 2007; Patel e Dickenson 2016). Additionally, studies have demonstrated that PGB induces neuroprotective effects by reducing oxidative stress and disrupting inflammatory processes in different pathological situations like sepsis, neuropathy, and retinopathy associated with diabetes (Sałat et al. 2016; Aslankoc et al. 2018; Cruz-Álvarez et al. 2018; Ali et al. 2019; Demir et al. 2021).

Hence, in the present study, we initially investigated whether non-diabetic and induced-T1DM animals exposed or not to a contextual fear conditioning session would perform distinctly, in a short- and longterm way, the behaviors related to the context-conditioned fear and anxiety. Next, we studied whether a single injection of PGB, before a fear memory extinction training, would be able to induce beneficial short and long-term - actions on fear extinction memory and anxiety-like responses. In the last set of experiments, we investigated in vitro and ex vivo a likely antioxidant activity of PGB. 


\section{Material And Methods}

\section{Animals}

Young male Wistar rats weighing between 180 - 220g from the Central Vivarium of the Federal University of Paraná were used. These animals were kept under controlled conditions, with temperature $22 \pm 2^{\circ} \mathrm{C}$, 12:12 light/dark cycle, with water and food (Nuvilab CR1 - Nuvital Nutrientes S/A) ad libitum. Four rats were kept per box $(60 \times 25 \times 15 \mathrm{~cm})$, with the shavings changed daily due to the polyuria induced by the diabetic condition. All experimental procedures were performed in accordance with the Brazilian Law for Animal Experimental Ethics and Care (11.794/8 October 2008) and was approved by the local Ethics Committee for the Use of Animals in the Biological Sciences Sector of the Federal University of Paraná (CEUA/BIO-UFPR, \#1131).All efforts were made to minimize the number of rats and their suffering.

\section{Drugs and Treatment protocol}

The following drugs were used: Streptozotocin (STZ, Cayman Chemicals, USA), Pregabalin (PGB, Pfizer,Brazil). STZ (60 mg/kg) was freshly dissolved in sodium citrate buffer (10 mM; pH 4.5) and administered intraperitoneally (i.p.) to induce T1DM. Citrate buffer was administered as a control for normoglycemic (NGL) groups.PGB (0,30, 100 and $300 \mathrm{mg} / \mathrm{kg}$, i.p.) was suspended in sterile $0.9 \%$ saline and prepared immediately before administration, in a volume of $2 \mathrm{~mL} / \mathrm{kg}$ of body weight. Dose of STZ was chosen based on previous studies from our group (de Morais et al. 2014; Gambeta et al. 2016; Pereira et al. 2018; de Souza et al. 2019; Chaves et al. 2020). Regarding PGB, route of administration and doses were taken from previous studies (Field et al. 2001; Zohar et al. 2008). The time of treatment (1 hour before being exposed to the apparatus) was defined based on pharmacokinetic characteristics, i.e. PGB reaches its peak plasma concentration after 1 hour of administration, bioavailability is approximately $90 \%$, regardless of dose and frequency of administration, and half-life of the molecule is about 6 hours (Buoli et al. 2017).

\section{Experimentaltype-1 diabetesmellitus(T1DM) induction}

T1DM was induced by a single intraperitoneal (i.p.) injection of STZ $(60 \mathrm{mg} / \mathrm{Kg})$ in overnight fasten rats (Chaves et al.2020). The diabetic condition was confirmed $72 \mathrm{~h}$ after the STZ injection using samples of about $5 \mu \mathrm{L}$ of blood from the tail vein added to test strips impregnated with glucose oxidase (Accu-Check Active $^{\mathrm{TM}}$, Roche). NGL animals received citrate buffer $(10 \mathrm{mM}, \mathrm{pH} 4.5$, equivalent volume). Only rats with blood glucose levels $\geq 250 \mathrm{mg} / \mathrm{dL}$ were considered diabetic and maintained in the study (about $80 \%$ of animals reached this parameter).

\section{Behavioral tests}

All behavioral tests were video recorded using camera Sony action cam $4 \mathrm{~K}$ for further analysis. The tests were carried out during the light phase (between 7:00 a.m. and 12:00 p.m.) in a sound-attenuated experimental room kept at $22 \pm 2^{\circ} \mathrm{C}$. The rats were acclimatized to the experimental room for at least 30 
min before each test. After each experimental session, the apparatus was cleaned with a $20 \%$ ethanol solution.

\section{Contextual Fear Conditioning (CFC)Test}

For the investigation of behaviors related to fear memory, the contextual fear conditioning (CFC) model was used(Ribeiro et al., 2020). The apparatus consisted of a rectangular chamber $(26 \times 31.5 \times 21 \mathrm{~cm}$; Insight, Ribeirão Preto, SP, Brazil). The bottom of the box consisted of small metal bars attached to an electrical stimulator which delivered foot shocks. The following steps were performed

- CFC session: This session was conducted by exposing the animal to the chamber (conditioned stimulus - CS) and after 30s they received 3 electrical footshocks (US; $1 \mathrm{~mA}$, lasting 3s), with 30s of intertrial interval. The animal remained in this chamber 30 s more before being returned to its home cage. - Extinction training session: 24 hours after, the animals were placed in the same chamber (CS) used during the CFC session, but they did not receive any US presentation. For20 minutes, the freezing time of the animals was evaluated in seconds (divided into 4 blocks of 5 min each).

- Extinction test 1: In the next day, the test was performed. For that, animals remained in the context (CS) for 3 minutes without the presentation of US and the time (in seconds) that the animal spent in freezing was quantified.

- Extinction test 2: The test was performed 7 days after the Extinction test 1, being this test exactly as the Extinction test 1.

The freezing behavior was used as an aversive conditioning index, and it was measured in seconds (s) and expressed as the percentage of total session time. Freezing behavior was considered when the animal presented a posture of complete immobility, except for breathing movements.

\section{Open-field test (OFT)}

The test was performed to evaluate the locomotor/exploratory activity according to Chaves et al. (2020). Thus, animals were placed in the center of open field apparatus that consisted of a rectangular wood arena $(40 \times 50 \times 63 \mathrm{~cm})$ with the floor divided into 9 rectangles situated in an isolated room and illuminated with an incandescent lamp(60 lux at the arena floor level). The number of crossed rectangles with the four paws was evaluated during $5 \mathrm{~min}$.

\section{Elevated plus-maze test (EPMT)}

The animals were exposed to the EPMT immediately after the Extinction test 2 (see section 2.4.1for details). The apparatus consisted of a wooden platform with 4 arms (2 open and 2 closed), raised $50 \mathrm{~cm}$ from the floor. There was a central area of $10 \mathrm{~cm}^{2}$ making the intersection between all the arms. The test was initiated by placing a rat on the central platform of the maze, facing a closed arm and the session had a duration of 5 minutes. The percentage of open arm entries (total amount of entries/number of 
open-arm entries $\times 100$ ) and the percent of time spent in the open arms served as the measure of anxiety. The frequency of entries in the closed arms was used as locomotor activity index (Cruz et al. 1994). Additionally, one ethological measure was added, the head dipping, which consist in the exploratory movement of head/shoulders over sides of the open arms and down towards the floor(Silva and Brandão 2000).

\section{2,2-diphenyl-1-picrylhydrazyl (DPPH) assay}

This assay was performed according to previous study (Pereira et al. 2018) to evaluate the potential antioxidant of PGB. It is used to evaluate the free radical scavenging activity of antioxidants. It is based on the principle that DPPH, upon accepting a hydrogen atom from a scavenger molecule (as an antioxidant), is reduced, and the purple color of the solution becomes yellow, concomitant with a decrease in absorbance(Mishra et al. 2012). The technique consisted of measuring the reactivity of PGB at different concentrations $(1,3,10,30,100 \mu \mathrm{g} / \mathrm{mL})$ mixed with $D P P H$ methanolic solution $(10 \mu \mathrm{g} / \mathrm{mL})$. Ascorbic acid solution $(50 \mu \mathrm{g} / \mathrm{mL})$ was used as the positive control, and distilled water was used as the negative control. Absorbance was measured at $517 \mathrm{~nm}$ using a multi-modal microplate reader (BioTek Synergy HT, BioTek Instruments, Highland Park, VT, USA).

\section{Evaluation of oxidative stress parameters}

Brain samples: The animals were euthanized by decapitation and the PFC and HIP were dissected. After dissection, the brain areas were frozen in liquid nitrogen, and stored at $-80^{\circ} \mathrm{C}$ until further analysis. The brain samples were homogenized in phosphate buffer $\mathrm{pH} 6.5$ (1:10). The homogenate was used to determination of the reduced glutathione (GSH) levels. To quantify lipid peroxidation (LPO) rate, a part of homogenate was centrifuged at $9000 \mathrm{~g}$ in a micro-high-speed refrigerated centrifuge (VS-15000 CFNII, Vision Scientific, Daejeon, South Korea) for $20 \mathrm{~min}$ and the supernatant was used to measure the LPO.

\section{Determination of the reduced glutathione (GSH) levels}

The GSH levels were measured by the method described by Sedlak \& Lindsay, (1968) and according to Pereira et al. (2018). Thus, $100 \mu \mathrm{L}$ of the homogenate were mixed with trichloroacetic acid ( $80 \mu \mathrm{L}$ of $12.5 \%$ purity) and centrifuged at 6000 rotations per minute (VS-15000 CFNII, Vision Scientific, Daejeon, South Korea) for $15 \mathrm{~min}$ at $4^{\circ} \mathrm{C}$. Then, $20 \mu \mathrm{L}$ of the clear supernatant was mixed with $280 \mu \mathrm{L}$ of Tris buffer ( $0.4 \mathrm{M}, \mathrm{pH} 8.9)$ and $5 \mu \mathrm{L}$ of 5,5'-dithiobis-(2-nitrobenzoic acid) in methanol. The absorbance of the reaction solution was measured at $415 \mathrm{~nm}$ in a microplate reader (BioTek Synergy HT, BioTek Instruments, Highland Park, VT, USA). The individual values were interpolated in a standard curve of GSH to verify the linearity of the reaction. The results are expressed as $\mu \mathrm{mol} / \mathrm{g}$ of tissue.

\section{Determination of the lipid peroxidation (LPO)levels}

The LPO levels was measured by the FOX-2 method described by Jiang et al. (1991) and according to Pereira et al. (2018).For that, $100 \mu \mathrm{L}$ of supernatant of PFC and HIP were homogenized in $100 \mu \mathrm{l}$ of methanol, vortexed, and centrifuged at 5000 rpm (VS-15000 CFNII, Vision Scientific, Daejeon, South 
Korea) for $5 \mathrm{~min}$ at $4^{\circ} \mathrm{C}$. Then, $100 \mu \mathrm{L}$ of the supernatant was added to $900 \mu \mathrm{L}$ of FOX2 reagent (Wolff's reagent; $4 \mathrm{mM} \mathrm{BHT,} 250 \mathrm{mM}$ FeSO4, $250 \mathrm{mM} \mathrm{H} 2 \mathrm{SO} 4$, and $100 \mathrm{mM}$ xylenol orange). The absorbance was measured at $560 \mathrm{~nm}$ in in a microplate reader (BioTek Synergy HT, BioTek Instruments, Highland Park, VT, USA). The results are expressed as [hydroperoxides] nmoL. $\mathrm{min}^{-1} / \mathrm{mg}$ of tissue.

\section{Experimental design}

In all experiments, the blood glucose was assessed 72 hours after the experimental induction of T1DM with STZ and at the end of the experiments, being this last measurement considered for data analysis. The weight gain considered in the analysis was the subtraction of the body weight (taken after the experiments) from that body weight taken immediately before the STZ injection.

Experiment 1: This experiment was carried out to evaluate the impact of CFC on the fear extinction memory (short and long-term) and on late anxiety-like responses in induced-T1DM (STZ) and normoglycemic (NGL) animals. The animals were divided into groups exposed or not exposed to the CFC, conditioned (Cond) and non-conditioned (N.Cond), respectively. Thus, four groups were formed: STZ/Cond $(n=10), S T Z / N . C o n d(n=9), N G L / C o n d(n=10)$, and NGL/N.Cond $(n=10)$.

On the 24th day after the induction of diabetes, part of the animals was submitted to the protocol of CFC and part of them, the N.Cond group, was exposed to the same context but they did not receive footshocks (US). On day 25, all animals were submitted to the extinction training session. Twenty-four hours after, they were evaluated in the same context (Extinction test 1). Seven days later, the Extinction test 2 was performed followed by the EPMT, on the day 33. Then, the body weight and blood glucose (BG) were checked one more time.

Experiment 2: In this experiment, we aimed to evaluate if one single injection of PGB before the fear memory extinction training would facilitate this acquisition. Moreover, if this single injection would induce a long-term effect on the extinction memory and would disrupt the late anxious-like responses of inducedT1DManimals (STZ). Hence, five groups - all subjected to CFC-treated with vehicle (VEH) or pregabalin (PGB) were formed: NGL/VEH ( $\mathrm{n}=7)$, STZ/VEH ( $\mathrm{n}=7)$, STZ/PGB $30 \mathrm{mg} / \mathrm{Kg}(\mathrm{n}=10)$, STZ/PGB $100 \mathrm{mg} / \mathrm{Kg}$ $(\mathrm{n}=10)$, and STZ/PGB $300 \mathrm{mg} / \mathrm{Kg}(\mathrm{n}=10)$.

On the 24th day after the induction of diabetes, animals were submitted to the protocol of CFC. On day 25 , one hour before the Extinction training session all animals received injection of PGB (different doses) or VEH. Twenty-four hours later, they were evaluated in the same context (Extinction test 1) and after seven days, the Extinction test 21 was performed followed by the EPMT (day 33th ). Then, the body weight and blood glucose (BG) were checked.

Experiment 3: Bearing in mind that higher doses of PGB can cause a sedative effect, this experiment was performed to investigate whether during the extinction training session (occurred in the Experiment 2) the animals treated with PGB were under a sedative effect. Thus, five groups - all subjected to contextual conditioned fear - treated with vehicle (VEH) or pregabalin (PGB) were formed: NGL/VEH ( $n=6)$, STZ/VEH $(\mathrm{n}=6)$, STZ/PGB $30 \mathrm{mg} / \mathrm{Kg}(\mathrm{n}=6)$, STZ/PGB $100 \mathrm{mg} / \mathrm{Kg}(\mathrm{n}=6)$, and STZ/PGB $300 \mathrm{mg} / \mathrm{Kg}(\mathrm{n}=7)$. 
On the 24th day after the induction of diabetes, animals were submitted to the protocol of CFC. In the next day, all animals received injection of PGB (different doses) or VEH and 1 hour after they were submitted to the OFT. Then, the body weight and blood glucose (BG) were checked one more time.

\section{Experiment 4}

This in vitro experiment was performed to evaluate the antioxidant potential of PGB, according to the method of Chen et al. (2018) with modifications. The

Experiment 5: This study was carried out to assess whether the PGB in its lowest dose ( $30 \mathrm{mg} / \mathrm{kg})$ and highest dose $(300 \mathrm{mg} / \mathrm{kg}$ ) would present a neuroprotective profile in induced-T1DM animals (STZ). Thus, on the 24th day after the induction of diabetes the animals were conditioned to the context with 3 footshocks (US), and in the next day (25th day) they received a single injection of VEH or PGB 1 hour before the extinction training session. The animals were euthanized immediately after this session of extinction and had HIP and CPF dissected for analysis of the GSH and LPO levels. For comparative purposes, these analyses were also performed in the HIP and PFC from NGL animals. The sample $n$ for each 4 groups formed for analysis in the HIP and PFC were: For HIP - NGL/VEH $(n=6), \operatorname{STZ/VEH~}(n=5)$, STZ/PGB $30 \mathrm{mg} / \mathrm{Kg}(\mathrm{n}=7)$, and STZ/PGB $300 \mathrm{mg} / \mathrm{Kg}(\mathrm{n}=7)$. For PFC - NGL/VEH ( $\mathrm{n=6}), \mathrm{STZ/VEH}(\mathrm{n}=5)$, STZ/PGB $30 \mathrm{mg} / \mathrm{Kg}(\mathrm{n}=7)$, and STZ/PGB $300 \mathrm{mg} / \mathrm{Kg}(\mathrm{n}=7)$.

\section{Statistical analysis}

The Shapiro-Wilk normality test was initially used to ensure that the data met the criteria for performing parametric tests. When the criteria were met, the results were reported as the mean \pm standard error of the mean (SEM). For the experiment 1, two-way analysis of variance (ANOVA) with or without repeated measures (RM) was performed. To analyze the data obtained from extinction training session we considered as independent variables the different groups and the time (blocks of time) being the time the RM. In the other experimental sessions, the condition of the animals (NGL or STZ) and the procedure (Cond and N.Cond) were considered as independent variables. Freezing time was the dependent variable. For the experiment2, two-way analysis of variance (ANOVA)with or without repeated measures (RM) was used. To analyze extinction training data, we considered the different groups and the time as independent variables, being the time a RM. The freezing time was used as the dependent variable. In the other experimental sessions and for the experiment 3 (OFT) and 4 (oxidative stress analysis), the one-way ANOVA was applied.

When appropriate, Bonferroni post-hoc test was used to perform multiple comparisons and the differences were considered statistically significant when $p<0.05$. All the tests were carried out using the GraphPad Prism program (version 8, San Diego, CA, USA).

\section{Results}

\section{Experiment 1:}


- Short- and long-term effectsof CFC on the fear extinction memory in induced-T1DM and NGL animals.

Two-way ANOVA with RM showed a significant difference when the data from extinction training session were analyzed (Fig. $1 A)$ - difference for blocks of time $[F(1,35)=159.9, p<0.05]$ as well as groups $[F(3$, $35)=175, p<0.05]$, and interaction between these variables $[F(3,35)=47.82, p<0.05]$. The CFC procedure increased the freezing time of NGL and induced-T1DM (STZ) animals when compared with its respective N.Cond groups $(p<0.05)$. Also, the freezing time was reduced in both Cond groups $-S T Z$ and NGL - when comparing the 4 th block with the 1 st block $(p<0.05)$. However, the freezing time was even more pronounced in STZ animals (Cond group), when compared with NGL (Cond group) at 4th block ( $<$ 0.05).

In the analysis of the freezing time during the extinction test 1 session (Fig. 1B), two-way ANOVA showed statistically significant difference for the condition of animals (NGL and STZ) $[F(1,35)=100.1, p<0.05]$ as well for the procedure $[F(1,35)=205.8, p<0.05]$, and interaction between these variables $[F(1,35)=$ 94.27, $p>0.05]$. When evaluated the extinction test 2 session (Fig. 1C), two-way ANOVA showed statistically significant difference for the condition of animals (NGL and STZ) $[F(1,36)=43.79, p<0.05]$ for the procedure $[F(1,35)=41.47, p<0.05]$, and interaction between these variables $[F(1,35)=41.23, p$ $>0.05]$. Bonferroni post-hoc test showed that in the session 1 the NGL Cond presented an increase in freezing time compared to NGL (N.Cond) group $(p<0.05)$. Also, an increase in freezing time was observed in the STZ (Cond) group in these two sessions when compared to its respective N.Cond group $(p<0.05)$ and also to NGL (Cond) $(p<0.05)$.

\section{- Long-term effects of CFC procedure followed byfear extinction memory training on anxiety-like responses in induced-T1DM and NGL animals.}

- In the analysis of the anxiety-like behavior (Fig. 2A-D), the two-way ANOVA showed significant difference for the condition of animals (NGL and STZ) when time $[F(1,35)=43.06, p<0.05$; Fig. 2A] and number of entries $[F(1,35)=38.96, p<0.05$; Fig. $2 B E]$ in the open arms were evaluated, as well head dipping $[F(1,35)=11.97, p<0.05$; Fig. $2 C]$ and entries in the closed arms $[F(1,35)=69.54, p<$ 0.05 ; Fig. 2D]. For the procedure factor, ANOVA showed difference only when entries in the open arms $[F(1,35)=29.95, p<0.05$; Fig. $2 B]$ was analyzed and interaction between the two factors (condition and procedure) when time in the open arms was evaluated $[F(1,35)=5.09, p<0.05$; Fig. 2A].

Bonferroni post-hoc test showed that among NGL animals, compared to N.Cond group, the Cond group presented a decrease on the time spent in the open arms and number of entries in these same arms $(p<$ 0.05), indicative of anxiogenic-like response. Comparing NGL and STZ (Cond groups), STZ animals exhibited a more pronounced decrease on time spent in the open arms and number of entries in these arms $(p<0.05)$, suggestive of a more expressive anxiogenic-like effect. When head dipping was evaluated, Bonferroni test showed a decrease in this behavior in STZ animals (Cond and N.Cond groups) 
compared to NGL (N.Cond) $(p<0.05)$.Also, a significant difference $(p<0.05)$ in the entries in the closed arms was observed in STZ animals, compared to NGL animals, independent of the procedures $(p<0.05)$.

When weight gain and glycemia were analyzed, one-way ANOVA showed a significant difference between the groups (NGL N.Cond, NGL Cond, STZ N.Cond and STZ Cond) [weight gain: $F(3,35)=149.8, p<0.05$; glycemia: $F(3,35)=203.1, p<0.05]$. Bonferroni post-hoc test showed that STZ animals (Cond and N.Cond) presented a decrease in the weight gain compared to NGL (Cond and N.Cond) $(p<0.05)$. The same was observed regarding to glycemia, i.e. STZ animals (Cond and N.Cond) presented an increase in the glycemia compared to NGL (Cond and N.Cond) $(p<0.05)$ (see Table S1 - supplementary material).

\section{Experiment 2}

\section{-Short- and long-term effects of a single injection of PGB on the acquisition of fear extinction memory in induced-T1DM animals.}

As can be seen in Fig. 3 when analyzed the extinction training data (Fig. 3A), two-way ANOVA with RM showed statistical difference between the groups $[F(4,36)=14.81, p<0.05]$,difference in time $[F(1,36)=$ $104.8 p<0.05]$ and interaction between these variables $[F(4,36)=9.737 p<0.05]$. Bonferroni post-hoc analysis showed a significant reduction in freezing among all the first PGB block groups compared to STZ/VEH 1st block group $(p<0.05)$. The NGL, STZ and PGB300 groups showed a significant difference in relation to their respective 1 st block group $(p<0.05)$.

In extinction test1 (Fig. 3B) and extinction test 2 (Fig. 3C) sessions, one-way ANOVA showed, respectively, significant effect of the treatment between STZ groups $[F(4,36)=24.36, p<0.05]$ and $[F(4,36)=59.83, p$ $<0.05$ ].The Bonferroni post-hoc analysis showed in extinction test 1 (Fig. 3B) that all groups treated with PGB presented a significant reduction in freezing $(p<0.05)$, as well as in extinction test 2 (Fig. $3 C)$, showing that the PGB effect was long-lasting duration. Furthermore, the injection of PGB 300 normalized the freezing behavior, not differing statistically from the NGL/VEH group.

\section{- Long-term effects of a single injection of PGB on anxiety-like responses in T1DM animals exposed previously to the CFC procedure and fear extinction training.}

In the EPMT, one-way ANOVA showed that the treatment was able to change the percentage of time spent in the open arms $[F(4,36)=5.322, p<0.05$; Fig. 4A], the percentage of entries in the open arms $[F(4,36)$ $=11.89, p<0.05 ;$ Fig. 4B], frequency of head dipping $[F(4,36)=5.661, p<0.05 ;$ Fig. $4 C]$, but did not change the total number of entries in the closed arms $[F(4,36)=3.283, p>0.05$; Fig. 4D]. Bonferroni post-hoc test showed that STZ animals treated with PGB 300 presented a significant reduction of the anxious-like behavior when compared to STZ/VEH during time and entries in the open arms, and frequency of head dipping $(p<0.05)$. Moreover, regarding to anxiety, STZ groups treated with PGB30 and/or PGB100 presented an increase in the percentage of time spent and entries in the open arms ( $p$ $<0.05)$. 
One-way ANOVA showed a significant difference among the groups when weight gain $[F(4,36)=119.5, p$ $<0.05]$ and blood glucose $[F(4,36)=56.92, p<0.05]$ were analyzed. Bonferroni post-hoc test showed that all STZ animals presented a significant reduction in the weight gain $(p<0.05)$ and an increase in the glycemia compared to NGL animals $(p<0.05)$ (see Table $S 1$ - supplementary material) and these parameters in STZ animals were not altered by any treatment employed in this study.

\section{Experiment 3 -Effect of a single injection of PGB on the locomotor activity ofinduced-T1DM animals submitted to the OFT.}

As shown in Fig. 5, one-way ANOVA test showed difference between NGL and STZ groups in the number of crossings in the OFT $[F(4,26)=30.67, \mathrm{p}<0.05]$. The Bonferroni post-hoc test showed that STZ animals treated with $P G B 300$ presented a significant decrease in the number of crossings compared to groups STZ/VEH and NGL/VEH $(p<0.05)$.

\section{Experiment 4 - Evaluation of PGB antioxidant activity: DPPH assay.}

As shown in Fig. 6, one-way ANOVA test showed difference between groups in DPPH $[F(6,14)=431.5, p$ $<0.0001$ ]. The Bonferroni post-hoc test showed that ascorbic acid and PGB (all concentrations) were able to induce an antioxidant activity $(\mathrm{p}<0.05)$.

\section{Experiment 5 -Effects of a single injection of PGB on parameters related to the oxidative stress, evaluated in the HIP and PFC of induced-T1DM animals.}

As shown in Fig. 7, one-way ANOVA test showed difference between treatments in the expression of LPO in the PFC $[F(3,23)=8.631, p<0.05]$ and in the $\operatorname{HIP}[F(3,23)=8.544, p<0.05]$, in addition to the difference between the GSH groups in the PFC $[F(3,23)=6.811, p<0.05]$ and $\operatorname{HIP}[F(3,23)=4.062, p<$ 0.05]. The Bonferroni post-hoc test showed that STZ/VEH animals presented a significant increase of LPO (PFC and HIP) and decrease of GSH (PFC and HIP), when compared with NGL/VEH $(p<0.05)$. While animals treated with PGB $300 \mathrm{mg} / \mathrm{kg}$ showed a significant reduction in the LPO in PFC and HIP compared to the STZ/VEH group ( $<<0.05)$, a significant increase of GSH in the PFC was also observed when PGB 30 and $300 \mathrm{mg} / \mathrm{kg}$ was administered.

\section{Discussion}

The main findings of the present study are that STZ animals show a persistent impairment in extinguishing a fear memory associated with delayed fear sensitization demonstrated by the increased anxiety-like response. Interestingly, a single injection of PGB in these STZ animals just before fear extinction training was able to facilitate fear extinction memory and alleviate anxiety-like behavior. These effects were observed in short and long-term (9 days later) periods. In addition, our data indicate that the beneficial effects induced by PGB may be related, at least partially, to its antioxidant activity demonstrated in the HIP and PFC. 
As already observed (Ikeda et al. 2015, 2021; de Souza et al. 2019; Ribeiro et al. 2020), our data confirmed that STZ animals present a greater fear response and a difficulty in extinguishing fear memory when compared to NGL animals (Fig. 1), indicating an overconsolidation of this fear memory. However, this is the first study to show the persistence of this fear memory in animals with induced-T1DM when reexposed in the same context after 1 week later (Fig. 1C) indicating how strong or dysfunctional are the mechanisms associated with this type of learning/memory. In this same direction, clinical studies point out a higher prevalence of PTSD in adults with T1DM (Renna et al. 2016), along with a positive association between the trauma of war and an increase in the incidence of T1DM in children and adolescents (Zung et al. 2012). The presence of severe and moderate PTSD symptoms has also been demonstrated in children (8-18 years) diagnosed with T1DM (Auxéméry 2012).

Regarding anxiety, STZ animals (not exposed to the same context of CFC) showed a more exacerbated anxiety-like response (decrease in the time spent and the number of entries in the open arms and decrease in the head dipping frequency) when compared to NGL animals - conditioned or not (Fig. 2A-C). These findings reinforce previous reports showing that these induced-T1DM animals present a more pronounced anxious-like behavior when compared to NGL animals (de Morais et al. 2014; Gambeta et al. 2016; Rebolledo-Solleiro et al. 2016; de Souza et al. 2019). Concerning NGL animals, even after acquiring extinction memory, they exhibited a more pronounced later anxiety-like behavior than non-conditioned NGL animals (Fig, 2A-D). This result is not surprising since other evidence show that previous stress may induce a response related to a fear sensitization or even to a generalization of fear memory, which depends on several aspects, such as external factors including the type and intensity of aversive stimulation, early-life stress, as well as the saliency of particular elements in the environment (Korte e De Boer 2003; Asok et al. 2019).

The next block of experiments was designed based on two main premises: 1. Induced-T1DM rats have an overconsolidation of fear memory, in addition to demonstrating the persistence of this memory and anxiety-like behavior, and 2. the use of PGB is approved for diabetic neuropathic pain, and its use has been successfully employed for treating more severe states of anxiety disorders and also PTSD (Pande et al. 2003; Pohl et al. 2005; Garakani et al. 2020). Our results revealed that all doses of PGB were able to decrease the freezing time when submitted to the extinction tests 1 and 2 (Fig. 3B-C), indicating that the drug facilitated the acquisition of this extinction memory, being this effect of long-lasting. Interestingly, the highest dose of PGB (300 mg/ $\mathrm{kg}$ ) was able to restore the behavior of the STZ animals, equating it with NGL animals (Fig. 3B-C). Regarding the delayed effect on the anxiety-like response, PGB (two highest doses) restored the most expressive anxious-like behavior of STZ animals, while only the highest dose (300 mg/kg) was able to induce a significant improvement in the behavior of head dipping (Fig. 4C). That is, the beneficial effect of PGB on fear memory seems not to be dissociated from its anxiolytic-like effect.

Although we found a sedative effect caused by the acute injection of PGB in the dose of $300 \mathrm{mg} / \mathrm{kg}$ (during extinction training) as demonstrated in the OFT (Fig. 5), the animals showed significant and consistent effects on fear extinction memory (Fig. 3B-C) and anxiety-like responses (Fig. 4A-C) in the subsequent tests in which they were not under this acute effect of PGB, once the half-life of PGB is 
around 6 hours (Buoli et al. 2017). Thus, this sedative effect during the extinction training session did not impair the acquisition of fear extinction memory and the anxiolytic-like effect. In addition, lower doses of PGB (30 mg/ $\mathrm{kg}$ and $100 \mathrm{mg} / \mathrm{kg}$ ) also showed effects in reducing freezing and improving anxiety, without inducing a sedative effect (Fig. 5).

At this point, we could mention as a limitation of the present study the fact that the best effects have been achieved after the administration of the highest dose of PGB. In this sense, it is known that the clinical efficacy dosage of PGB in GAD, for example, is $150-600 \mathrm{mg} /$ day, and the frequently reported side effects (drowsiness and dizziness) are dose-dependent (Baldwin et al. 2015). In double-blind, placebocontrolled trials the adverse events during treatment with PGB were considered mild and moderate (Mann et al. 2014). However, in a recent meta-analysis study, Onakpoya et al. (2019) demonstrated that despite being effective for the treatment of diseases such as diabetic peripheral neuropathy and post-herpetic neuralgia, many patients discontinue the treatment due to adverse effects. Furthermore, some studies have reported cases of increased heart failure, peripheral edema, and also Addiction. All these effects seem to be related to the chronic use of PGB (Robert Lee Page et al. 2008; Grosshans et al. 2010; Dobrea et al. 2012; Gahr et al. 2013; Aldemir et al. 2015). According to Buoli et al. (2017) at the highest doses of $P G B$, there is an increase of the enzyme responsible for the synthesis of GABA (L-Glutamic acid decarboxylase), and although PGB does not directly bind to GABA-A or GABA-B receptors, these receptors may be more active due to increased GABA levels (Buoli et al. 2017). Also, at the highest dose, PGB may bind to $a 2 \delta-2$ subunit, in addition to the $a 2 \delta-1$ subunit, which in the brain is concentrated in the cerebellum and partially correlates with GABAergic neurons (Barclay et al. 2001; Li et al. 2011). Thus, the adverse effects observed at the highest doses of PGB may be related preferentially to the unspecific effects of PGB on other sites of action. Here, if we think in translational terms, it is important to bear in mind what would be the risk and benefit of the treatment for the patient, and this response should be linked to the type of treatment and the dosage used. For example, in the present study, despite the most effective dose being the highest dose, which could cause a series of unwanted effects in continuous treatment, the PGB application was unique and possibly not associated with the consequences of adverse effects.

The International guidelines consider PGB along with the selective serotonin reuptake inhibitors (paroxetine, sertraline, escitalopram), and serotonin noradrenaline reuptake inhibitors as first-line options for treating GAD (Pande et al. 2003; Buoli et al. 2017; Greenblatt e Greenblatt 2018). Moreover, both openlabel and randomized, double-blind, placebo-controlled studies have demonstrated the efficacy of PGB, being in many of these studies superior to antidepressants and benzodiazepines depending on the parameter evaluated (for a review see Frampton 2014; Baldwin et al. 2015 and Buoli et al. 2017). Regarding treating PTSD with PGB, although studies are still limited, a retrospective clinical study of 290 burned service members revealed that PGB or gabapentin did not affect the development of PTSD (Fowler et al. 2012). However, in a randomized clinical trial, Baniasadi et al. (2014) demonstrated that PGB was able to reduce emotional symptoms of PTSD related to combat in a group of 18 male patients (non-diabetics) who received $300 \mathrm{mg} /$ day of PGB for a period of 6 weeks. 
In preclinical studies, it is evident that depending on the experimental protocol, such as the condition of the animal (including the animal's condition or the type of stress), doses used, and the time of treatment and its duration, the results are quite different. For example, Zohar et al. (2008) showed, in non-diabetic animals, that PGB (at the same doses used in the present study) induced beneficial short-term effects on behavioral responses (anxiety-like response) in animals exposed to traumatic stimuli (predator urine scent). However, the anxiolytic-like effect of PGB was observed only in these animals pre-exposed to predator urine scent, and not in that one's not pre-exposed. Also, these effects were not observed after 30 days of the PGB injection. Differently, Valdivieso et al. (2018) did not observe any beneficial effect after PGB treatment on anxiety-like behavior in previously stressed (restraint and tail shock) non-diabetic animals. Nevertheless, they used a smaller dose of PGB $(10 \mathrm{mg} / \mathrm{kg})$.

Of particular interest for the present study, all these changes related to an exacerbated behavioral response observed in induced-T1DM animals have been related to the diabetic encephalopathy (Gupta et al. 2014; Prabhakar et al. 2015; da Silva Dias et al. 2016; Zanoveli et al. 2016; Aswar et al. 2017; Wang et al. 2019; Chaves et al. 2020). Thus, in the following experiments (Experiment 4 and 5) we investigated whether PGB would present an antioxidant activity per se and whether this single injection of PGB, 1 hour before the fear extinction memory training, would exert an antioxidant action by improving indirect oxidative stress parameters in the HIP and PFC from STZ rats. Our data demonstrated that PGB (in all concentrations - Fig. 6) presented an antioxidant activity by showing a lower absorbance level compared to the negative control (water). Also, PGB was able to reduce the increased LPO levels of STZ animals (HIP and PFC - highest dose) and to increase the reduced GSH levels (PFC). These changes on reduced GSH and LPO levels in these brain regions of STZ animals (Fig. 7) have been previously demonstrated (Pitocco et al. 2010; Pereira et al. 2018; de Souza et al. 2019; Réus et al. 2019). Regarding LPO, it is known that high levels of LPO are attributed to the increase of reactive oxygen species (Siba et al. 2017); in addition, it affects cell integrity only when antioxidant mechanisms are no longer able to cope with the generation of free radicals (Anwer et al. 2012). The GSH is the main antioxidant in the brain (Kanazawa et al. 2016) and it protects the cellular system against the toxic effects of LPO. Thereby, according to Sharma et al. (2016), this decline in the level of GSH in induced-T1DM animals may be due to excessive free radical generation, exposure to high glucose levels. In addition, it has been proposed that antioxidant compounds may play an important role in improving the dysregulations reported here, especially in brain regions such as PFC and HIP, linked to memory/learning and emotional processing (Venturini et al. 2010; de Morais et al. 2014; Pereira et al. 2018; Banagozar Mohammadi et al. 2019; de Souza et al. 2019).

In this sense and reinforcing our data after PGB treatment, we have already observed in this animal model of T1DM that prolonged treatment with other antioxidant compounds, the vitamin E or gallic acid, improved the dysfunctional processing of fear memory and/or anxiety-like responses from these inducedT1DM animals along with an improvement on oxidative stress related-parameters in these same brain areas (de Morais et al. 2014; Pereira et al. 2018; de Souza et al. 2019). This antioxidant action of PGB has already been demonstrated in STZ animals (Sałat et al. 2016; Demir et al. 2021). For example, Sałat et al. (2016) evaluated the effects of the single injection of PGB in STZ mice on contextual memory (-not related to fear) and oxidative stress parameters. The authors reported that although PGB in a smaller 
dose $(10 \mathrm{mg} / \mathrm{kg})$ was not able to attenuate T1DM-induced memory impairments, the PGB did not aggravate learning deficits of these diabetic mice. However, this dose was able to disrupt some markers of oxidative stress. More recently, Demir et al. (2021) demonstrated that prolonged treatment with PGB ( $50 \mathrm{mg} / \mathrm{kg} /$ day for 8 weeks) was able to reduce LPO, improve antioxidant capacity with a significant increase in superoxide dismutase and protect cells against apoptosis.

It is important to note that in the present study, the lowest dose of PGB (30 mg/kg), which was the dose that changed more discreetly the behavioral parameters evaluated in the present study, was able to increase only the reduced level of GSH in the PFC. Thus, we can speculate that the antioxidant action of PGB may not be the only mechanism responsible for the beneficial action of PGB on fear memory and anxiety-like response in these induced-T1DM animals. In this regard, it is not surprising that PGB induces neuroprotective effects through other mechanisms, including an anti-inflammatory action by disrupting the expression of several inflammatory markers (Sałat et al. 2016; Aslankoc et al. 2018; Cruz-Álvarez et al. 2018; Ali et al. 2019).

Taken together, a single injection of PGB in induced-T1DM rats in a specific time window - before a fear memory extinction training session - facilitates the acquisition of fear extinction memory in the short- and long-term, being this effect additionally associated with late improvement on anxiety-like behavior. All these short- and long-term beneficial actions of PGB may be associated with neuroprotective mechanisms of PGB, including its antioxidant action in brain areas like PFC and HIP. Despite the need for further investigations, the data are quite interesting and highlight the potential of PGB for future translational investigations, taking into account the cost-benefit of PGB as a facilitator or adjuvant in the process of extinction of the traumatic memories, in addition to anxiolytic effects.

\section{Declarations}

Acknowledgments - Coordenação de Aperfeiçoamento de Pessoal de Nível Superior - Brasil (CAPES Finance Code 001) and Conselho Nacional de Desenvolvimento Científico e Tecnologico (CNP). AHB de Lima Silva and DR Radulski are recipients of CAPES fellowships.

Funding and disclosure - This study was supported by Brazilian grants from Conselho Nacional de Desenvolvimento Científico e Tecnologico (CNPq; 303863/2020-0), which had no other role in the design of the study, collection and analysis of data, and decision to submit the paper for publication. JMZ is a recipient of fellowship awards from Conselho Nacional de Desenvolvimento Científico e Tecnológico (CNPq, Brazil - 1A).

Data availability- Data sharing not applicable to this article as no datasets were generated or analyzed in the current study

Declarations - The article is original and has been written by the stated authors who are all aware of its content and approve its submission. This article has not been published previously and it is not under 
consideration for publication elsewhere, in whole or in part. The authors declare that there are no financial or other relationships that might lead to a conflict of interest of the present article.

Contributors - All authors contributed to the study conception and design. Material preparation, data collection and analysis were performed by Janaina Menezes Zanoveli, Alvaro Henrique Bernardo de Lima Silva, Debora Rasec Radulski, Gabriela Saidel Pereira and Alexandra Acco. The first draft of the manuscript was written by Alvaro Henrique Bernardo de Lima Silva and Janaina Menezes Zanoveli and all authors commented on previous versions of the manuscript. All authors read and approved the final manuscript.

Compliance with ethical standards - All experiments were conducted in accordance with the rules and legislation contained by the UFPR Animal Research Ethics Committee (CEUA number \#1131).

\section{References}

1. Ahmed A, Zeng G, Jiang D et al (2019) Time-dependent impairments in learning and memory in Streptozotocin-induced hyperglycemic rats. Metab Brain Dis 34:1431-1446. https://doi.org/10.1007/s11011-019-00448-7

2. Aldemir E, Altintoprak AE, Coşkunol H (2015) Pregabalin dependence: A case report. Turk Psikiyatr Derg 26:217-220. https://doi.org/10.2174/1574886309666141022101956

3. Ali SA, Zaitone SA, Dessouki AA, Ali AA (2019) Pregabalin affords retinal neuroprotection in diabetic rats: Suppression of retinal glutamate, microglia cell expression and apoptotic cell death. Exp Eye Res 184:78-90. https://doi.org/10.1016/j.exer.2019.04.014

4. Anwer T, Sharma M, Pillai KK, Khan G (2012) Protective effect of Withania somnifera against oxidative stress and pancreatic $\beta$-cell damage in type 2 diabetic rats. Acta Pol Pharm - Drug Res 69:1095-1101

5. Aslankoc R, Savran M, Ozmen O, Asci S (2018) Hippocampus and cerebellum damage in sepsis induced by lipopolysaccharide in aged rats - Pregabalin can prevent damage. Biomed Pharmacother 108:1384-1392. https://doi.org/10.1016/j.biopha.2018.09.162

6. Asok A, Kandel ER, Rayman JB (2019) The neurobiology of fear generalization. Front Behav Neurosci 12. https://doi.org/10.3389/fnbeh.2018.00329

7. Aswar U, Chepurwar S, Shintre S, Aswar M (2017) Telmisartan attenuates diabetes induced depression in rats. Pharmacol Reports 69:358-364. https://doi.org/10.1016/j.pharep.2016.12.004

8. Auxéméry (2012) [Posttraumatic stress disorder (PTSD) as a consequence of the interaction between an individual genetic susceptibility, a traumatogenic event and a social context]. Encephale 38:373380

9. Baldwin DS, Den Boer JA, Lyndon G et al (2015) Efficacy and safety of pregabalin in generalised anxiety disorder: A critical review of the literature. J Psychopharmacol 29:1047-1060. https://doi.org/10.1177/0269881115598411 
10. Banagozar Mohammadi A, Torbati M, Farajdokht F et al (2019) Sericin alleviates restraint stress induced depressive- and anxiety-like behaviors via modulation of oxidative stress, neuroinflammation and apoptosis in the prefrontal cortex and hippocampus. Brain Res 1715:47-56. https://doi.org/10.1016/j.brainres.2019.03.020

11. Baniasadi M, Hosseini G, Bordbar MRF et al (2014) Effect of pregabalin augmentation in treatment of patients with combat-related chronic posttraumatic stress disorder: A randomized controlled trial. J Psychiatr Pract 20:419-427. https://doi.org/10.1097/01.pra.0000456590.12998.41

12. Barclay J, Balaguero N, Mione M et al (2001) Ducky mouse phenotype of epilepsy and ataxia is associated with mutations in the Cacna2d2 gene and decreased calcium channel current in cerebellar Purkinje cells. J Neurosci 21:6095-6104. https://doi.org/10.1523/jneurosci.21-1606095.2001

13. Bludau S, Bzdok D, Gruber $O$ et al (2016) Medial prefrontal aberrations in major depressive disorder revealed by cytoarchitectonically informed voxel-based morphometry. Am J Psychiatry 173:291298. https://doi.org/10.1176/appi.ajp.2015.15030349

14. Bremner JD, Randall P, Scott TM et al (1995) MRI-based measurement of hippocampal volume in patients with combat- related posttraumatic stress disorder. Am J Psychiatry 152:973-981. https://doi.org/10.1176/ajp.152.7.973

15. Buchberger B, Huppertz $H$, Krabbe $L$ et al (2016) Symptoms of depression and anxiety in youth with type 1 diabetes: A systematic review and meta-analysis. Psychoneuroendocrinology 70:70-84. https://doi.org/10.1016/j.psyneuen.2016.04.019

16. Buoli M, Caldiroli A, Serati M (2017) Pharmacokinetic evaluation of pregabalin for the treatment of generalized anxiety disorder. Expert Opin Drug Metab Toxicol 13:351-359. https://doi.org/10.1080/17425255.2017.1281247

17. Chaves YC, Genaro K, Stern CA et al (2020) Two-weeks treatment with cannabidiol improves biophysical and behavioral deficits associated with experimental type-1 diabetes. Neurosci Lett 729:135020. https://doi.org/10.1016/j.neulet.2020.135020

18. Cohen H, Geva AB, Matar MA et al (2008) Post-traumatic stress behavioural responses in inbred mouse strains: Can genetic predisposition explain phenotypic vulnerability? Int J Neuropsychopharmacol 11:331-349. https://doi.org/10.1017/S1461145707007912

19. Cruz-Álvarez LE, de la, Zúñiga-Romero Á, Huerta-Cruz JC et al (2018) Antiallodynic interaction and motor performance of the pregabalin/thioctic acid and pregabalin/a-tocopherol combinations in neonatal streptozotocin-induced diabetic rats. Drug Dev Res 79:362-369. https://doi.org/10.1002/ddr.21473

20. Cruz APM, Frei F, Graeff FG (1994) Ethopharmacological analysis of rat behavior on the elevated plus-maze. Pharmacol Biochem Behav 49:171-176. https://doi.org/10.1016/0091-3057(94)90472-3

21. da Silva Dias IC, Carabelli B, Ishii DK et al (2016) Indoleamine-2,3-Dioxygenase/Kynurenine Pathway as a Potential Pharmacological Target to Treat Depression Associated with Diabetes. Mol Neurobiol 53:6997-7009. https://doi.org/10.1007/s12035-015-9617-0

Page $17 / 28$ 
22. de Morais H, de Souza CP, da Silva LM et al (2014) Increased oxidative stress in prefrontal cortex and hippocampus is related to depressive-like behavior in streptozotocin-diabetic rats. Behav Brain Res 258:52-64. https://doi.org/10.1016/j.bbr.2013.10.011

23. de Souza CP, Gambeta E, Stern CAJ, Zanoveli JM (2019) Posttraumatic stress disorder-type behaviors in streptozotocin-induced diabetic rats can be prevented by prolonged treatment with vitamin E. Behav Brain Res 359:749-754. https://doi.org/10.1016/j.bbr.2018.09.008

24. Demir CF, Balduz M, Taşcı İ, Kuloğlu T (2021) Protective effect of pregabalin on the brain tissue of diabetic rats. Diabetol Int 12:207-216. https://doi.org/10.1007/s13340-020-00476-0

25. Dobrea C, Buoli M, Arici $C$ et al (2012) Tolerability and use in co-administration of pregabalin in affective patients: A 6-month prospective naturalistic study. Expert Opin Drug Saf 11:893-899. https://doi.org/10.1517/14740338.2012.720971

26. dos Santos MAB, Ceretta LB, Réus GZ et al (2014) Anxiety disorders are associated with quality of life impairment in patients with insulin-dependent type 2 diabetes: A case-control study. Rev Bras Psiquiatr 36:298-304. https://doi.org/10.1590/1516-4446-2013-1230

27. Field MJ, Oles RJ, Singh L (2001) Pregabalin may represent a novel class of anxiolytic agents with a broad spectrum of activity. Br J Pharmacol 132:1-4. https://doi.org/10.1038/sj.bjp.0703794

28. Finnerup NB, Attal N, Haroutounian S et al (2015) Pharmacotherapy for neuropathic pain in adults: A systematic review and meta-analysis. Lancet Neurol 14:162-173. https://doi.org/10.1016/S14744422(14)70251-0

29. Fowler M, Garza TH, Slater TM et al (2012) The relationship between gabapentin and pregabalin and posttraumatic stress disorder in burned servicemembers. J Burn Care Res 33:612-618. https://doi.org/10.1097/BCR.0b013e31823dc710

30. Frampton JE (2014) Pregabalin: A review of its use in adults with generalized anxiety disorder. CNS Drugs 28:835-854. https://doi.org/10.1007/s40263-014-0192-0

31. Gahr M, Franke B, Freudenmann RW et al (2013) Concerns about pregabalin: Further experience with its potential of causing addictive behaviors. J Addict Med 7:147-149. https://doi.org/10.1097/ADM.0b013e3182872718

32. Gambeta E, de Souza CP, de Morais H, Zanoveli JM (2016) Reestablishment of the hyperglycemia to the normal levels seems not to be essential to the anxiolytic-like effect induced by insulin. Metab Brain Dis 31:563-571. https://doi.org/10.1007/s11011-015-9770-1

33. Garakani A, Murrough JW, Freire RC et al (2020) Pharmacotherapy of Anxiety Disorders: Current and Emerging Treatment Options. https://doi.org/10.3389/fpsyt.2020.595584. Front Psychiatry 11:

34. Greenblatt HK, Greenblatt DJ (2018) Gabapentin and Pregabalin for the Treatment of Anxiety Disorders. Clin Pharmacol Drug Dev 7:228-232. https://doi.org/10.1002/cpdd.446

35. Grosshans M, Mutschler J, Hermann D et al (2010) Pregabalin abuse, dependence, and withdrawal: A case report. Am J Psychiatry 167:869. https://doi.org/10.1176/appi.ajp.2010.09091269

36. Gupta D, Radhakrishnan M, Kurhe Y (2014) Insulin reverses anxiety-like behavior evoked by streptozotocin-induced diabetes in mice. Metab Brain Dis 29:737-746. 
https://doi.org/10.1007/s11011-014-9540-5

37. Ikeda H, Ikegami M, Kai M, Kamei J (2015) Cannabinoid functions in the amygdala contribute to conditioned fear memory in streptozotocin-induced diabetic mice: Interaction with glutamatergic functions. Exp Neurol 269:233-241. https://doi.org/10.1016/j.expneurol.2015.04.012

38. Ikeda H, Yamamoto S, Kamei J (2021) Increase in brain I-lactate enhances fear memory in diabetic mice: Involvement of glutamate neurons. Brain Res 1767:147560.

https://doi.org/10.1016/j.brainres.2021.147560

39. Jacobson AM, Ryan CM, Cleary PA et al (2011) Biomedical risk factors for decreased cognitive functioning in type 1 diabetes: An 18 year follow-up of the Diabetes Control and Complications Trial (DCCT) cohort. Diabetologia 54:245-255. https://doi.org/10.1007/s00125-010-1883-9

40. Jiang ZY, Woollard ACS, Wolff SP (1991) Lipid hydroperoxide measurement by oxidation of Fe2+ in the presence of xylenol orange. Comparison with the TBA assay and an iodometric method. Lipids 26:853-856. https://doi.org/10.1007/BF02536169

41. Kanazawa LKS, Vecchia DD, Wendler EM et al (2016) Quercetin reduces manic-like behavior and brain oxidative stress induced by paradoxical sleep deprivation in mice. Free Radic Biol Med 99:7986. https://doi.org/10.1016/j.freeradbiomed.2016.07.027

42. Korte SM, De Boer SF (2003) A robust animal model of state anxiety: Fear-potentiated behaviour in the elevated plus-maze. Eur J Pharmacol 463:163-175. https://doi.org/10.1016/S00142999(03)01279-2

43. Li Z, Taylor CP, Weber M et al (2011) Pregabalin is a potent and selective ligand for a $2 \delta-1$ and a $2 \delta-2$ calcium channel subunits. Eur J Pharmacol 667:80-90. https://doi.org/10.1016/j.ejphar.2011.05.054

44. Lustman PJ, Clouse RE (2005) Depression in diabetic patients: The relationship between mood and glycemic control. J Diabetes Complications 19:113-122. https://doi.org/10.1016/j.jdiacomp.2004.01.002

45. MacQueen GM, Campbell S, McEwen BS et al (2005) Course of Illness, Hippocampal Function, and Hippocampal Volume in Major Depression. Focus (Madison) 3:146-155. https://doi.org/10.1176/foc.3.1.146

46. Mann D, Liu J, Chew ML et al (2014) Safety, tolerability, and pharmacokinetics of pregabalin in children with refractory partial seizures: A phase 1, randomized controlled study. Epilepsia 55:19341943. https://doi.org/10.1111/epi.12830

47. Matsunaga Y, Negishi T, Hatakeyama A et al (2016) Impairment of synaptic development in the hippocampus of diabetic Goto-Kakizaki rats. Int J Dev Neurosci 53:58-67. https://doi.org/10.1016/j.ijdevneu.2016.07.004

48. Mishra T, Das AP, Sen A (2012) Phytochemical Screening and In-vitro Antioxidant Profiling of Solvent Fractions of Canna edulis Ker Gawler. Free Radicals Antioxidants 2:13-20. https://doi.org/10.5530/ax.2012.2.5 
49. Moulton CD, Pickup JC, Ismail K (2015) The link between depression and diabetes: The search for shared mechanisms. Lancet Diabetes Endocrinol 3:461-471. https://doi.org/10.1016/S22138587(15)00134-5

50. Muriach M, Flores-Bellver M, Romero FJ, Barcia JM (2014) Diabetes and the brain: Oxidative stress, inflammation, and autophagy. Oxid Med Cell Longev 2014:. https://doi.org/10.1155/2014/102158

51. Onakpoya IJ, Thomas ET, Lee JJ et al (2019) Benefits and harms of pregabalin in the management of neuropathic pain: A rapid review and meta-analysis of randomised clinical trials. BMJ Open 9. https://doi.org/10.1136/bmjopen-2018-023600

52. Pande AC, Crockatt JG, Feltner DE et al (2003) Pregabalin in generalized anxiety disorder: A placebocontrolled trial. Am J Psychiatry 160:533-540. https://doi.org/10.1176/appi.ajp.160.3.533

53. Patel R, Dickenson AH (2016) Mechanisms of the gabapentinoids and a2 $\delta-1$ calcium channel subunit in neuropathic pain. Pharmacol Res Perspect 4. https://doi.org/10.1002/prp2.205

54. Pereira MM, de Morais H, dos Santos Silva E et al (2018) The antioxidant gallic acid induces anxiolytic-, but not antidepressant-like effect, in streptozotocin-induced diabetes. Metab Brain Dis 33:1573-1584. https://doi.org/10.1007/s11011-018-0264-9

55. Pitocco D, Zaccardi F, Di Stasio E et al (2010) Oxidative stress, nitric oxide, and diabetes. Rev Diabet Stud 7:15-25. https://doi.org/10.1900/RDS.2010.7.15

56. Pohl RB, Feltner DE, Fieve RR, Pande AC (2005) Efficacy of pregabalin in the treatment of generalized anxiety disorder: Double-blind, placebo-controlled comparison of BID versus TID dosing. J Clin Psychopharmacol 25:151-158. https://doi.org/10.1097/01.jcp.0000155820.74832.b0

57. Prabhakar V, Gupta D, Kanade P, Radhakrishnan M (2015) Diabetes-associated depression: The serotonergic system as a novel multifunctional target. Indian J Pharmacol 47:4-10. https://doi.org/10.4103/0253-7613.150305

58. Rajput R, Gehlawat P, Gehlan D et al (2016) Prevalence and predictors of depression and anxiety in patients of diabetes mellitus in a tertiary care center. Indian J Endocrinol Metab 20:746-751. https://doi.org/10.4103/2230-8210.192924

59. Rebolledo-Solleiro D, Araiza LFO, Broccoli L et al (2016) Dopamine D1 receptor activity is involved in the increased anxiety levels observed in STZ-induced diabetes in rats. Behav Brain Res 313:293301. https://doi.org/10.1016/j.bbr.2016.06.060

60. Renna CP, Boyer BA, Prout MF, Scheiner G (2016) Posttraumatic Stress Related to Hyperglycemia: Prevalence in Adults with Type I Diabetes. J Clin Psychol Med Settings 23:269-284. https://doi.org/10.1007/s10880-016-9463-x

61. Réus GZ, Carlessi AS, Silva RH et al (2019) Relationship of oxidative stress as a link between diabetes mellitus and major depressive disorder. Oxid Med Cell Longev 2019:. https://doi.org/10.1155/2019/8637970

62. Ribeiro TO, Bueno-de-Camargo LM, Waltrick APF et al (2020) Activation of mineralocorticoid receptors facilitate the acquisition of fear memory extinction and impair the generalization of fear 
memory in diabetic animals. Psychopharmacology 237:529-542. https://doi.org/10.1007/s00213019-05388-9

63. Rickels K, Pollack MH, Feltner DE et al (2005) Pregabalin for treatment of generalized anxiety disorder: A 4-week, multicenter, double-blind, placebo-controlled trial of pregabalin and alprazolam. Arch Gen Psychiatry 62:1022-1030. https://doi.org/10.1001/archpsyc.62.9.1022

64. Robert Lee Page II, Cantu M, Lindenfeld J et al (2008) Possible heart failure exacerbation associated with pregabalin: Case discussion and literature review. J Cardiovasc Med 9:922-925. https://doi.org/10.2459/JCM.0b013e3282fb7629

65. Sałat K, Gdula-Argasińska J, Malikowska N et al (2016) Effect of pregabalin on contextual memory deficits and inflammatory state-related protein expression in streptozotocin-induced diabetic mice. Naunyn Schmiedebergs Arch Pharmacol 389:613-623. https://doi.org/10.1007/s00210-016-1230-x

66. Sedlak J, Lindsay RH (1968) Estimation of total, protein-bound, and nonprotein sulfhydryl groups in tissue with Ellman's reagent. Anal Biochem 25:192-205. https://doi.org/10.1016/00032697(68)90092-4

67. Sharma M, Gupta S, Singh K et al (2016) Association of glutathione-S-transferase with patients of type 2 diabetes mellitus with and without nephropathy. Diabetes Metab Syndr Clin Res Rev 10:194197. https://doi.org/10.1016/j.dsx.2016.06.006

68. Siba IP, Bortolanza M, Frazão Vital MAB et al (2017) Fish oil prevents rodent anxious states comorbid with diabetes: A putative involvement of nitric oxide modulation. Behav Brain Res 326:173-186. https://doi.org/10.1016/j.bbr.2017.03.008

69. Silva RCB, Brandão ML (2000) Acute and chronic effects of gepirone and fluoxetine in rats tested in the elevated plus-maze: An ethological analysis. Pharmacol Biochem Behav 65:209-216. https://doi.org/10.1016/S0091-3057(99)00193-8

70. Taylor CP, Angelotti T, Fauman E (2007) Pharmacology and mechanism of action of pregabalin: The calcium channel a2- $\delta$ (alpha2-delta) subunit as a target for antiepileptic drug discovery. Epilepsy Res 73:137-150. https://doi.org/10.1016/j.eplepsyres.2006.09.008

71. Tesfaye S, Wilhelm S, Lledo A et al (2013) Duloxetine and pregabalin: High-dose monotherapy or their combination? the "cOMBO-DN study" - A multinational, randomized, double-blind, parallel-group study in patients with diabetic peripheral neuropathic pain

72. Vaccarino V, Goldberg J, Magruder KM et al (2014) Posttraumatic stress disorder and incidence of type-2 diabetes: Aprospective twin study. J Psychiatr Res 56:158-164. https://doi.org/10.1016/j.jpsychires.2014.05.019

73. Valdivieso DA, Baughan TG, Canavati UM et al (2018) Effects of pregabalin on neurobehavior in an adult male rat model of PTSD. PLoS ONE 13. https://doi.org/10.1371/journal.pone.0209494

74. Venturini CD, Merlo S, Souto AA et al (2010) Resveratrol and red wine function as antioxidants in the central nervous system without cellular proliferative effects during experimental diabetes. Oxid Med Cell Longev 3. https://doi.org/10.4161/oxim.3.6.14741 
75. Wang XP, Ye P, Lv J et al (2019) Expression Changes of NMDA and AMPA Receptor Subunits in the Hippocampus in rats with Diabetes Induced by Streptozotocin Coupled with Memory Impairment. Neurochem Res 44:978-993. https://doi.org/10.1007/s11064-019-02733-4

76. Weisberg RB, Bruce SE, Machan JT (2002) Nonpsychiatric illness among primary care patients with trauma histories and posttraumatic stress disorder. Prim Care Companion J Clin Psychiatry 4:118

77. Zanoveli JM, de Morais H, da Dias IC et al (2016) Depression Associated with Diabetes: From Pathophysiology to Treatment. Curr Diabetes Rev 12:165-178.

https://doi.org/10.2174/1573399811666150515125349

78. Zohar J, Matar MA, Ifergane G et al (2008) Brief post-stressor treatment with pregabalin in an animal model for PTSD: Short-term anxiolytic effects without long-term anxiogenic effect. Eur Neuropsychopharmacol 18:653-666. https://doi.org/10.1016/j.euroneuro.2008.04.009

79. Zung A, Blumenfeld $O$, Shehadeh $\mathrm{N}$ et al (2012) Increase in the incidence of type 1 diabetes in Israeli children following the Second Lebanon War. Pediatr Diabetes 13:326-333. https://doi.org/10.1111/j.1399-5448.2011.00838.x

\section{Figures}
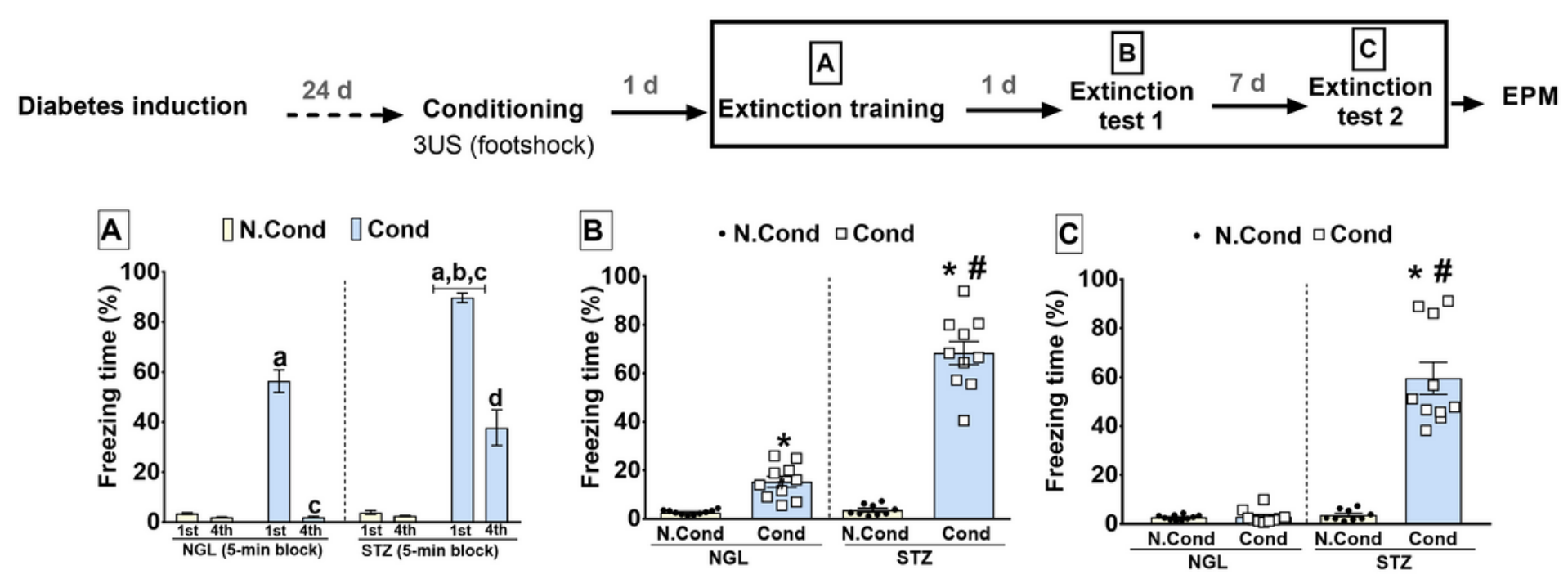

Figure 1

Short and long-term effects of contextual conditioned fear in normoglycemic (NGL) and STZ-induced T1DM (STZ) animals on fear extinction memory. The scheme above the graphs represents the experimental design used in this experiment. (A) Extinction training session of fear contextual memory; (B) Extinction test 1; (C) Extinction test 2. Values are expressed as mean \pm SEM ( $n=9-11 /$ group). a $p<$ 0.05 compared to 1 st block (NGL-N.Cond); bp $<0.05$ compared to 1 st block (STZ-N.Cond); $\mathrm{p}<0.05$ compared to 1 st block (NGL-Cond); $d p<0.05$ compared to other 4ths blocks; ${ }^{*} p<0.05$ when compared to all N.Cond groups; \# $\mathrm{p}<0.05$ compared to NGL-Cond group 

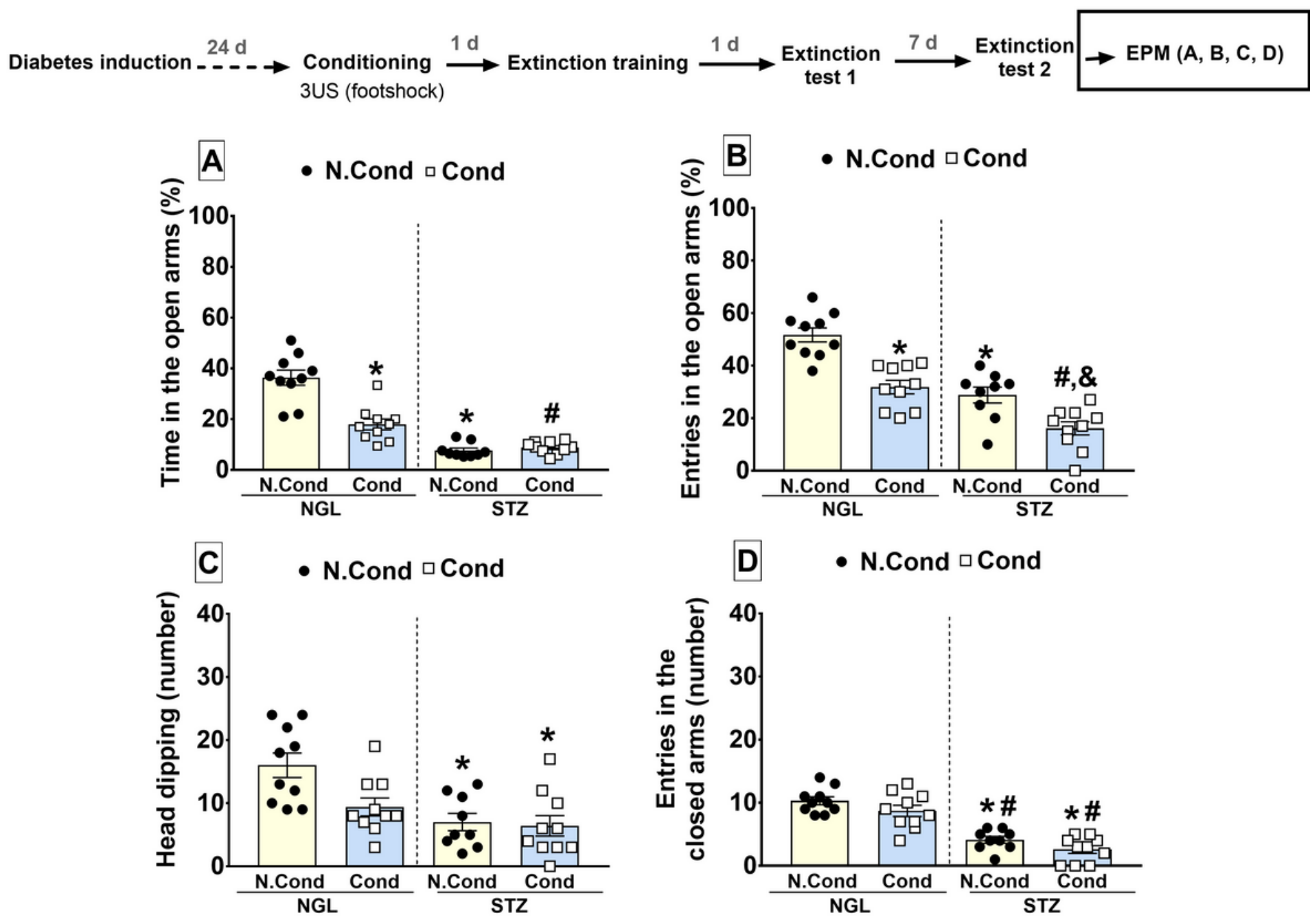

D $\quad$ N.Cond $\square$ Cond

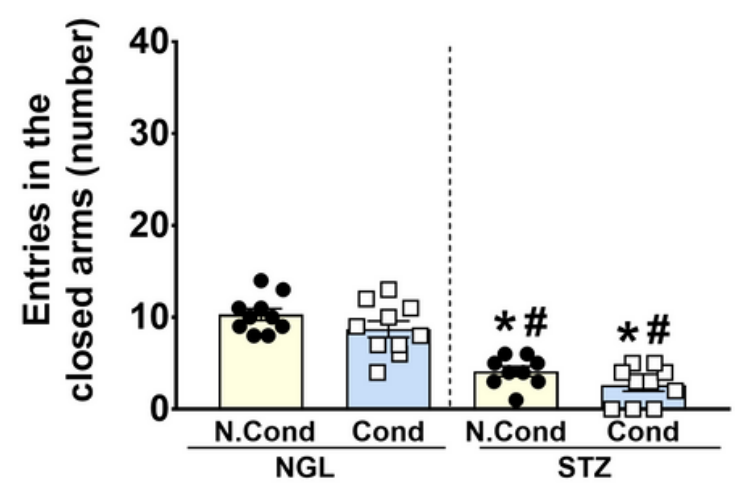

Figure 2

Long-term effects of contextual conditioned fear followed by fear extinction memory training in normoglycemic (NGL) and STZ-induced T1DM (STZ) animals on anxiety-like responses. The scheme above the graphs represents the experimental design used in this experiment. (A) \% of the time in open arms; (B) \% of the entries in the open arms, (C) frequency of head dipping; (D) number of entries in the closed arms. Values are expressed as mean \pm SEM ( $n=9-11 /$ group). ${ }^{*} p<0.05$ when compared to all N.Cond groups; \# $p<0.05$ compared to NGL-Cond group; \& $p<0.05$ compared to STZ-N.Cond group 


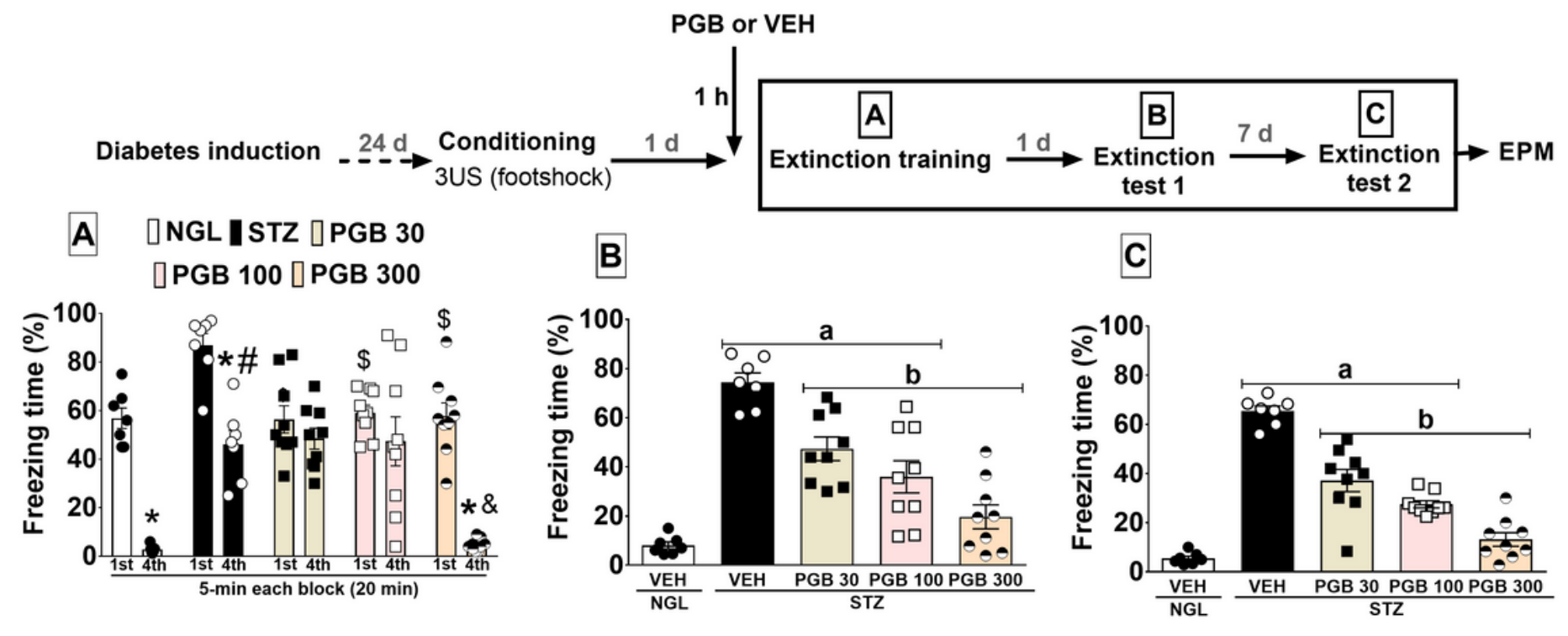

Figure 3

Short and long-term effects of a single injection of pregabalin (PGB) in STZ-induced T1DM (STZ) animals; or vehicle (VEH) - in normoglycemic (NGL) and STZ animals on the acquisition of fear extinction memory. The scheme above the graphs represents the experimental design used in this experiment. (A) Extinction training session of fear contextual memory; (B) Extinction test 1; (C) Extinction test 2. Values are expressed as mean \pm SEM ( $n=7-9 /$ group). ${ }^{*} p<0.05$ when compared to its respective 1 st block group; \# $\mathrm{p}<0.05$ compared to 4 th block (NGL) group; $\$ p<0.05$ compared to 1 st block (STZ) group; \& $p<0.05$ compared to 4 st block (STZ) group; a $p<0.05$ compared to NGL (VEH); b $p<0.05$ compared to STZ (VEH) 

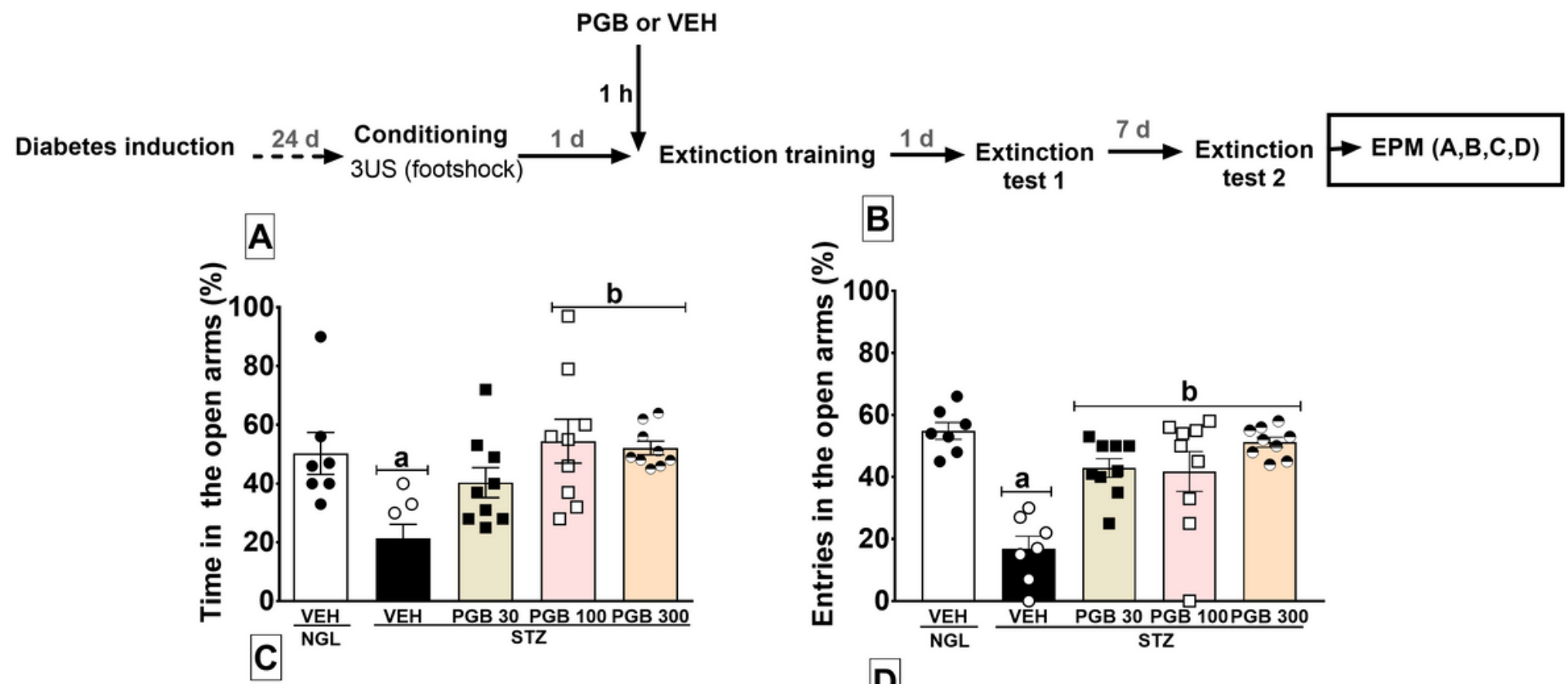

B

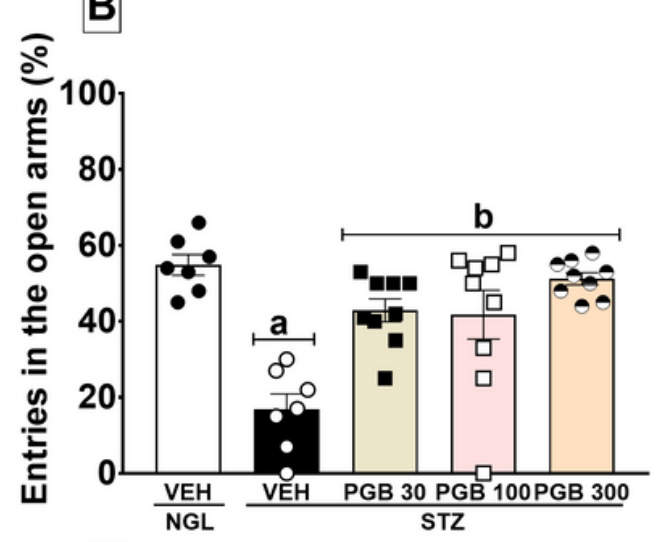

D
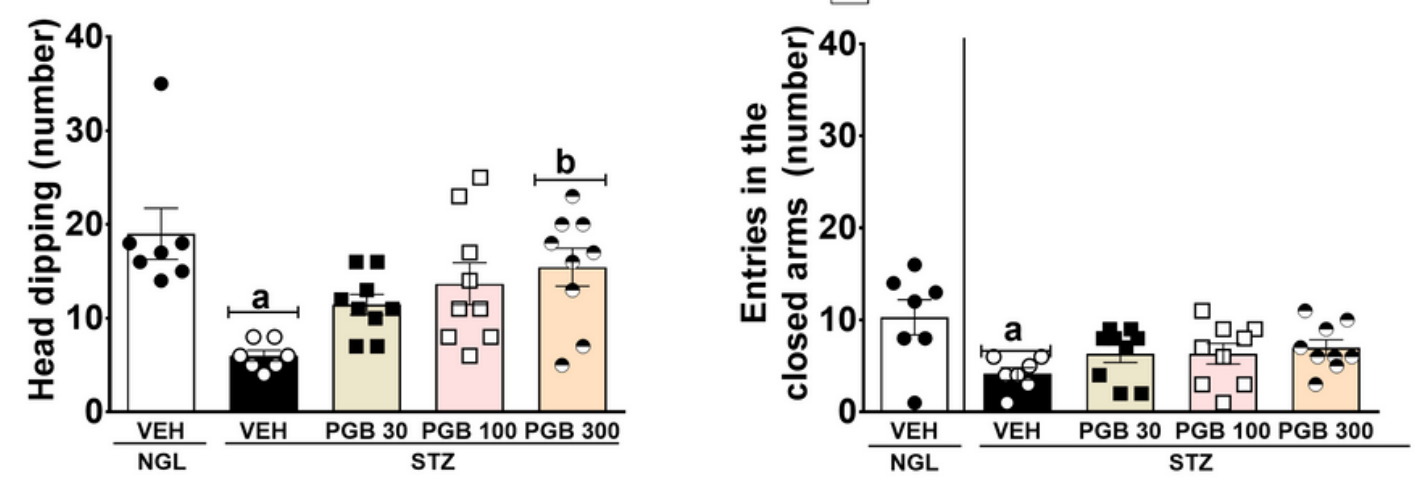

Figure 4

Long-term effects of a single injection of pregabalin (PGB) in STZ-induced T1DM (STZ) animals; or vehicle (VEH) - in normoglycemic (NGL) and STZ animals on anxiety-like responses. The scheme above the graphs represents the experimental design used in this experiment. (A) \% of the time in open arms; (B) $\%$ of the entries in the open arms, (C) frequency of head dipping; (D) number of entries in the closed arms. Values are expressed as mean \pm SEM ( $n=7-9 /$ group). a $p<0.05$ compared to NGL (VEH); $b \quad<<0.05$ compared to STZ (VEH) 


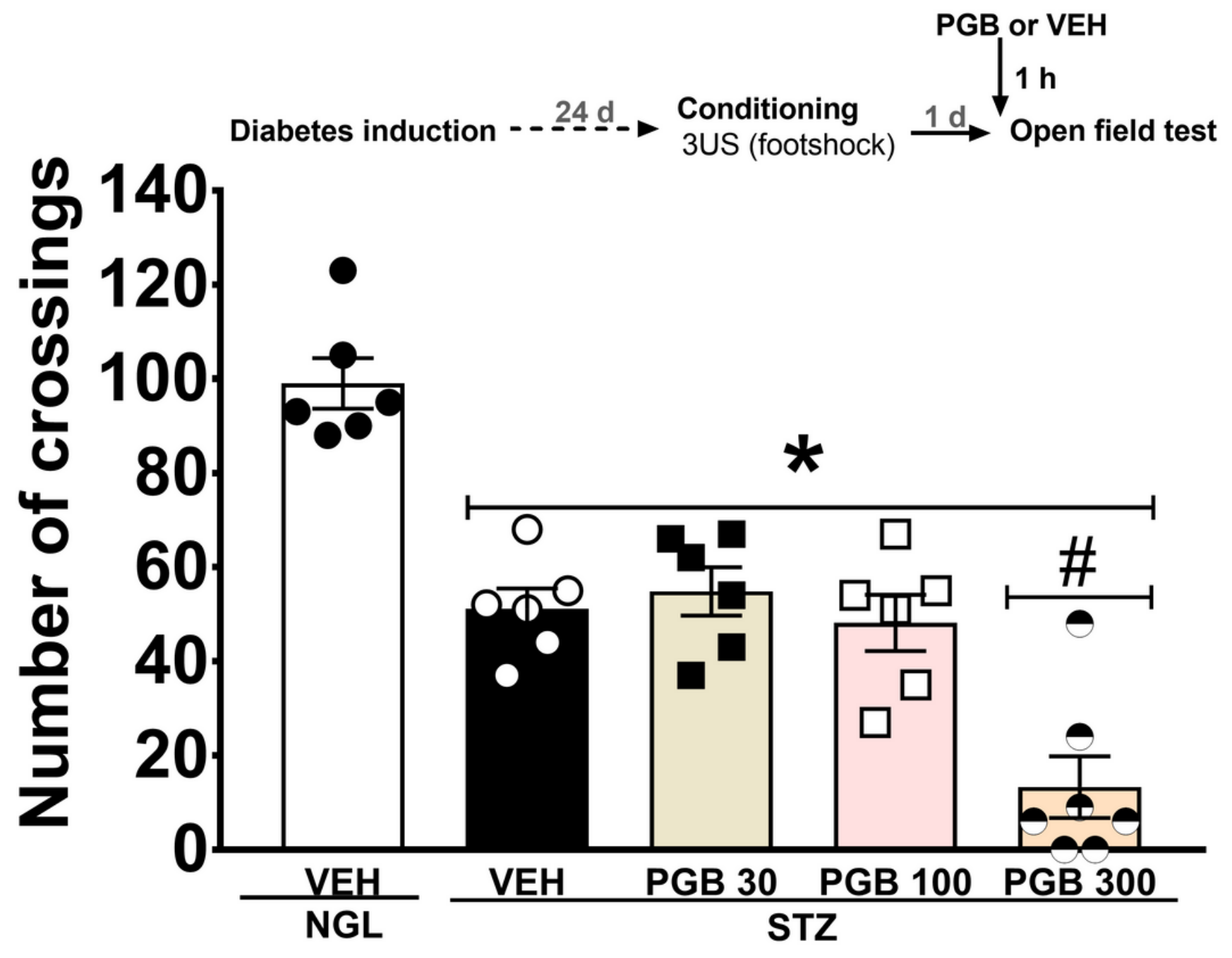

Figure 5

Effects of a single injection of pregabalin (PGB) in STZ-induced T1DM (STZ) animals, or vehicle (VEH) in normoglycemic (NGL) and STZ animals - on the number of crossings in the open field test. The scheme above graphs represents the experimental design used in this experiment. ${ }^{*} p<0.05$ when compared to NGL (VEH) group; \# $p<0.05$ compared to STZ (VEH) group 


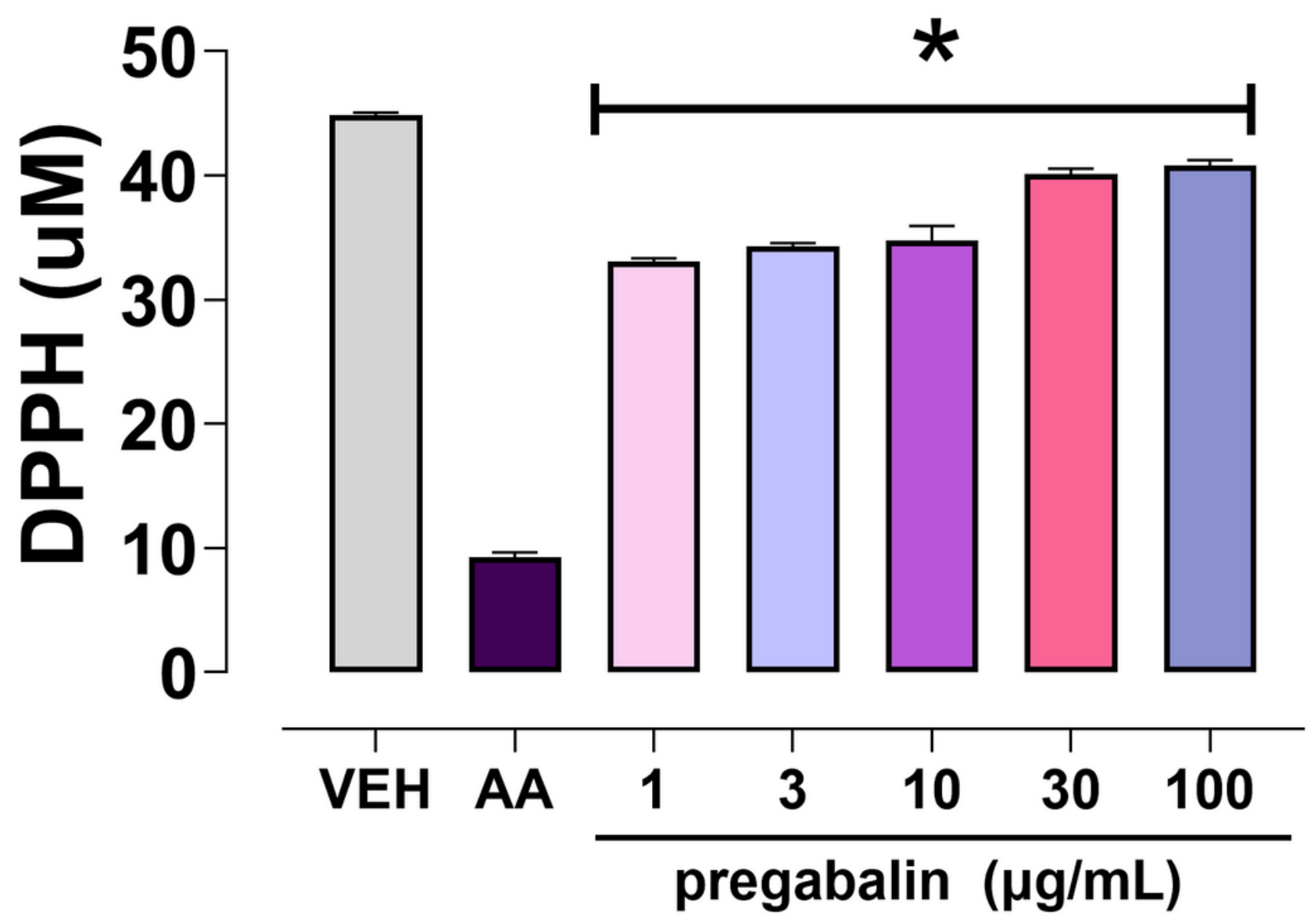

Figure 6

Effect of pregabalin, ascorbic acid (AA; positive control) and water (negative control) on free radical scavenging activity in the DPPH assay. Results are expressed as mean $\pm S E M ; n=3,{ }^{*} p<0.05$ compared to VEH 


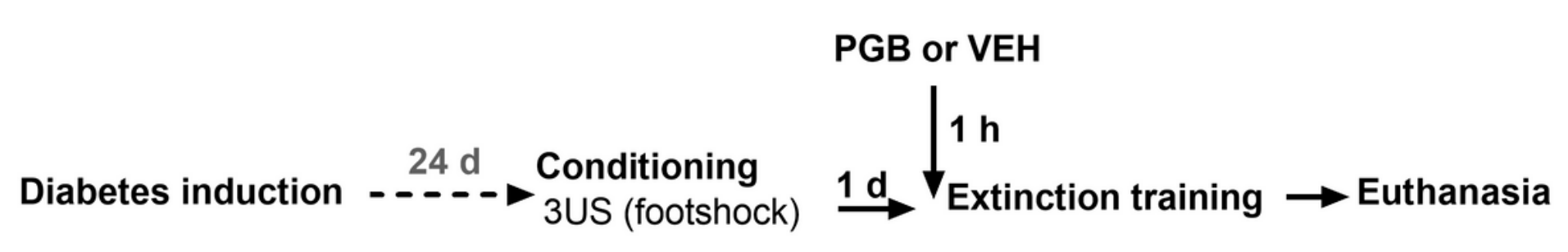

PREFRONTAL CORTEX
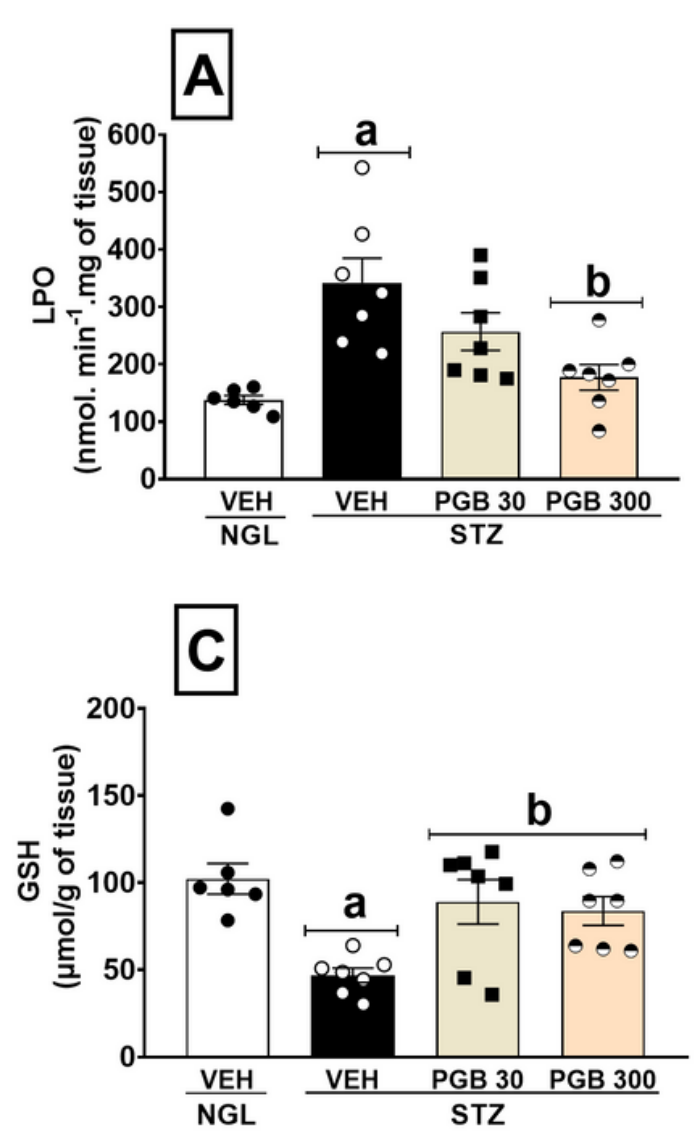

\section{HIPPOCAMPUS}

LPO

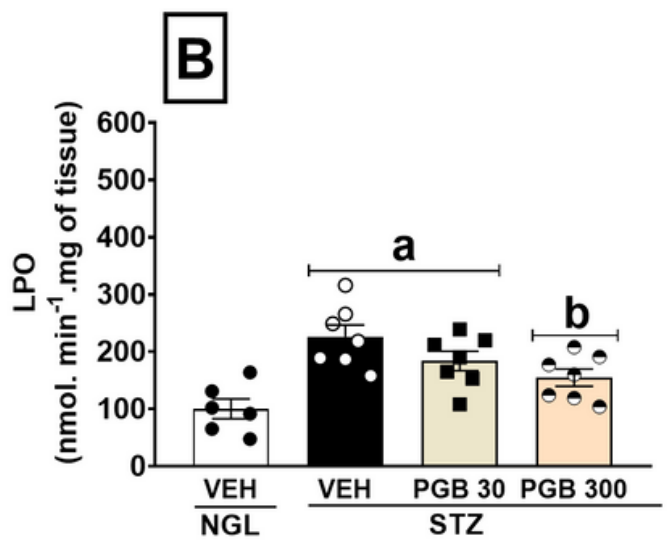

GSH

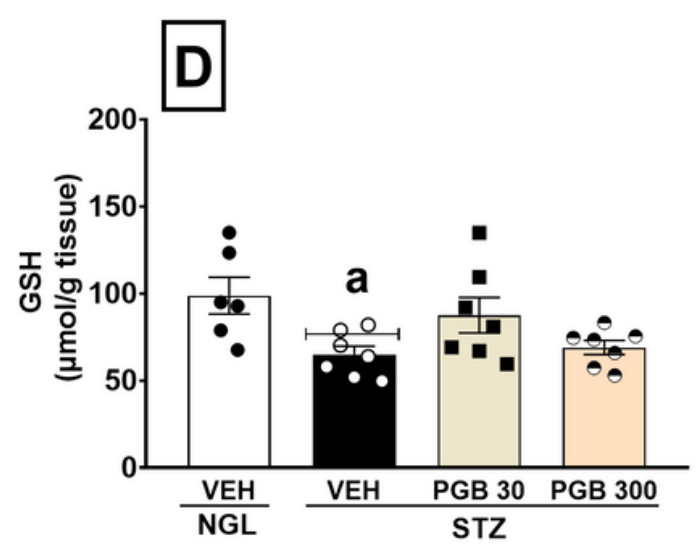

Figure 7

Effect of a single injection of pregabalin $(30,300 \mathrm{mg} / \mathrm{kg})$ or vehicle (VEH) in normoglycemic (NGL) or STZ-induced T1DM (STZ) animals on lipid peroxidation levels (LPO, panel A - prefrontal cortex and panel B - hippocampus) and reduced glutathione levels (GSH, panel C - prefrontal cortex and panel D hippocampus). Results are expressed as mean \pm SEM; $n=6-7$; $a p<0.05$ compared to NGL/VEH; $b p<$ 0.05 compared to STZ/VEH

\section{Supplementary Files}

This is a list of supplementary files associated with this preprint. Click to download.

- Supplementarymaterial.docx 